\title{
Chemical constituents of Cephalotaxus species
}

\author{
Anupam Bhattacharya, Virinder S. Parmar, Sunil K. Sharma, Smriti Trikha, Sanjay K. Singh and \\ Ashok K. Prasad ${ }^{*}$
}

Department of Chemistry, University of Delhi, Delhi-110 007, India

E-mail : minuashok@now-india.net.in Fax : 91-11-7667206

Manuscript received 30 July 200I, revised 20 May 2002, accepted 12 June 2002

The compounds isolated from Cephalotaxus species during the period 1930 to March 2002 have been reviewed; these have been listed showing their source(s).

\section{Introduction}

Cephalotaxus was once regarded as a genus of the family Taxaceae on the basis of morphology, anatomy, embryology and geographical distribution. But based upon the recent findings of chemical constituents of different species of this genus, the classification of the genus Cephalotaxus to a separate family, the Cephalotaxaceae of the order Coniferae has been supported ${ }^{1,2}$. Cephalotaxus, the only genus of this family consists of mainly eleven species, namely, $C$. drupacea (now known as $C$. harringtonia var. drupacea), C. follicularis, C. fortunei Hook. f., C. griffithii Hook,. f., $C$. hainanensis Linn., C. harringtonia (forbes) Koch., C. lanceolata, C. mannii Hook. f., C. oliveri Mast., C. sinensis Li. - and $C$. wilsoniana.

Cephalotaxus is native to Eastern Asia and plants of this genus are evergreen coniferous trees with yew like leaves or shrubs that grow in humid valleys or in forests at an elevation of 100-2000 m. Cephalotaxus species, commonly known as plum yew, are generally hard and ornamental, and succeed on a variety of soils; they can be propagated from seeds and cuttings. The plum yews can be grown as hedges and excellent screens. The three species, namely, $C$. griffithii Hook. f., C. harringtonia (or C. drupacea) and $C$. mannii Hook. f. occur in India, $C$. harringtonia also occurs in Japan and the remaining eight species alongwith $C$. mannii are indigenous to China ${ }^{3.4}$. Cephalotaxus species have also been reported to be used as traditional medicine, e.g. Shanjishan, a traditional Chinese medicine derived from bark and leafy branches of $C$. fortunei Hook $f$., is used as immunosuppresant and antineoplastic agent ${ }^{5}$.

Cephalotaxus species, in general, are mainly known for alkaloids; different alkaloids isolated from Cephalotaxus species have been reviewed earlier'. Several flavones, biflavones, taxanes, sterols, etc. have also been isolated from different Cephalotaxus species. In this review, we give an update and comprehensive compilation of different classes of compounds isolated from various species of Cephalotaxus, alongwith their biological activities. Various compounds isolated from eleven different Cephalotaxus species have been classified under the classes of alkaloids, biflavones, flavones, sterols and miscellaneous compounds and are listed in Tables 1-5.

\section{Alkaloids}

Wall et al. ${ }^{6}$ reported in 1954 the first phytochemical examination of the genus Cephalotaxus and showed the
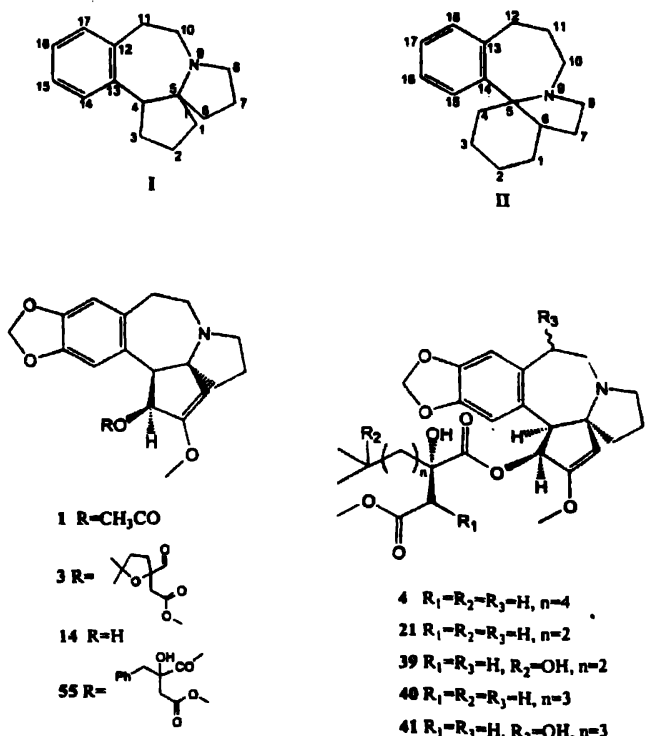

4 $R_{1}=R_{2}=R_{3}-H, n=4$. $21 R_{1}=R_{2}=R_{3}=H_{1} n=2$ $39 R_{1}=R_{3}=H_{1} R_{2}=O H, n=2$ $40 R_{1}=R_{2}=R_{3}-H_{1} n=3$ $41 R_{1}=R_{3}=H_{1} R_{2}=H_{,} n=3$ is $R_{1}=R_{2}=H_{1} R_{3}-\beta-O H, n=2$ $47 R_{1}=R_{2}=H, R_{3}=-\mathrm{OH}, \mathrm{a}=3$ 4s $R_{1}=R_{2}=H, R_{1}=\beta-O H, n=3$

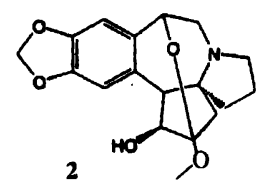
S6 $R_{1}=R_{2}=R_{3}=H, n=1$ 


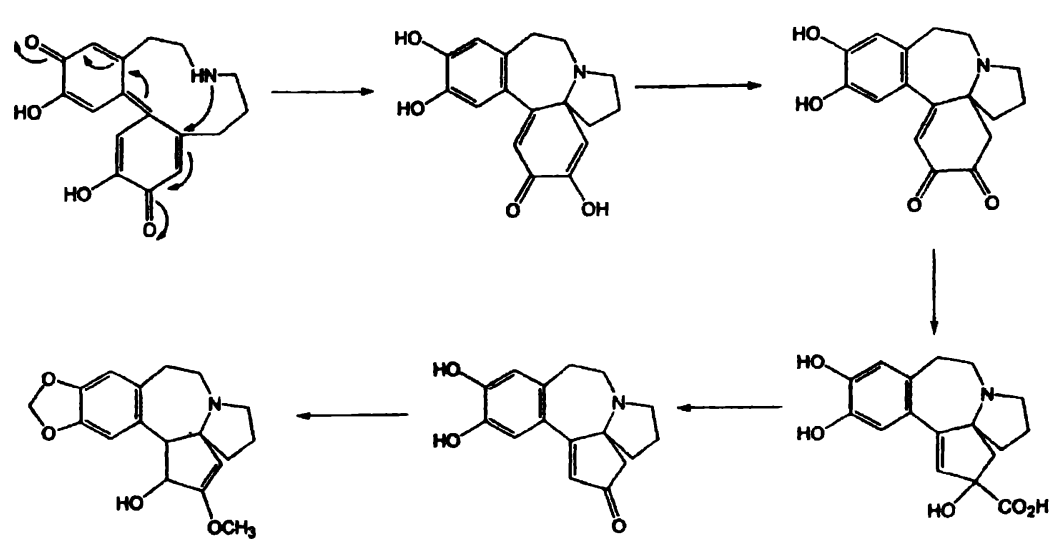

Scheme 1. Biosynthetic route suggested for the derivation of the cephalotaxine series of alkaloids.

presence of alkaloids in this genus. Paudler et al. ${ }^{7}$ isolated cephalotaxine (14), a crystalline alkaloid from $C$. harringtonia var. drupacea and $C$. fortunei. Two types of alkaloids, namely, cephalotaxine and homoerythrina
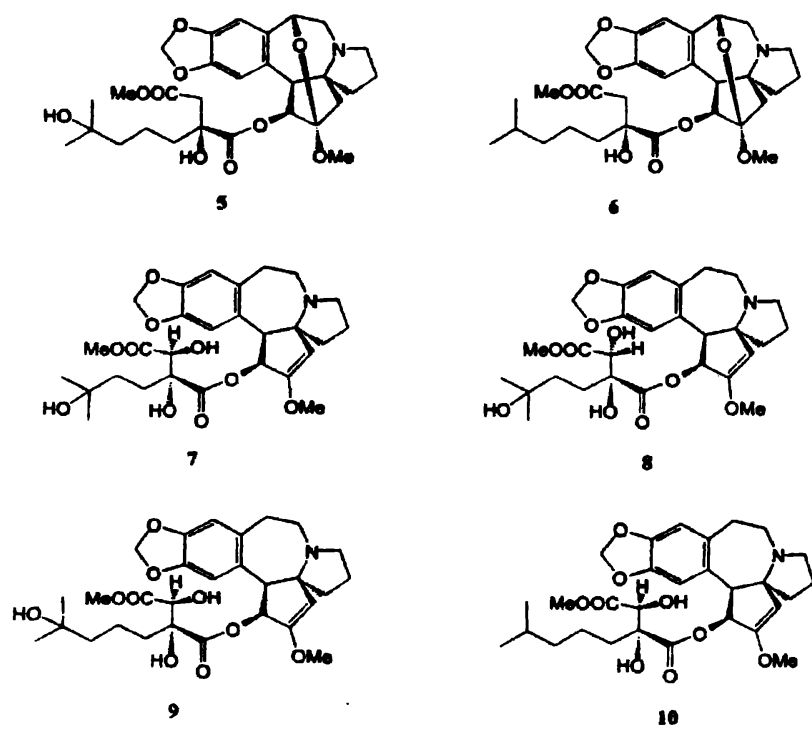

alkaloids based upon the skeletons I and II, respectively, are found in the genus Cephalotaxus. The homoerythrina alkaloids are present as minor alkaloids in this genus with the exception in $C$. wilsonian ${ }^{8}$. The occurrence of cephalotaxus and homoerythrina alkaloids in the same plant suggests that they may be formed through similar biogenetic routes (Scheme 1) ${ }^{9}$.

Cephalotaxine (14) accounts for nearly $50 \%$ of the total alkaloids present in Cephalotaxus plant extracts but it is inactive. Three antitumour alkaloids, all esters of cephalotaxine (14), i.e. harringtonine (39), homoharringtonine (41) and isoharringtonine (52), isolted from $C$. harringtonia have shown significant inhibitory activity against experimental lymphoid leukemia systems L1210 and $\mathrm{P} 388$ in mice ${ }^{10}$. Zhang et al. ${ }^{11}$ reported the inhibitory activity of harringtonine (39) against sarcoma 180 in mice and against nonlymphoblastic leukemia in clinical experiments. In clinical tests, harringtonine (39) appeared to be effective in treating acute and chronic myelocytic leukemia in human subjects ${ }^{12}$. Harringtonine (39) and homoharringtonine (41) had significant activities against P388 leukemia, L1210 leukemia and B16 melanoma. The therapeutic indexes $\left(\mathrm{LD}_{10}\right.$ and $\left.\mathrm{ED}_{50}\right)$ in $\mathrm{B} 16$ melanoma system $^{13}$ were 1.90 and 2.31 . The alkaloids isolated from $C$. fortunei were used for the treatment of lymphosarcoma, Hodgkin's disease, reticular cell sarcoma, lung cancer and for various kinds of leukemia and malignant tumors. The total alkaloids of $C$. sinensis and $C$. hainanensis showed

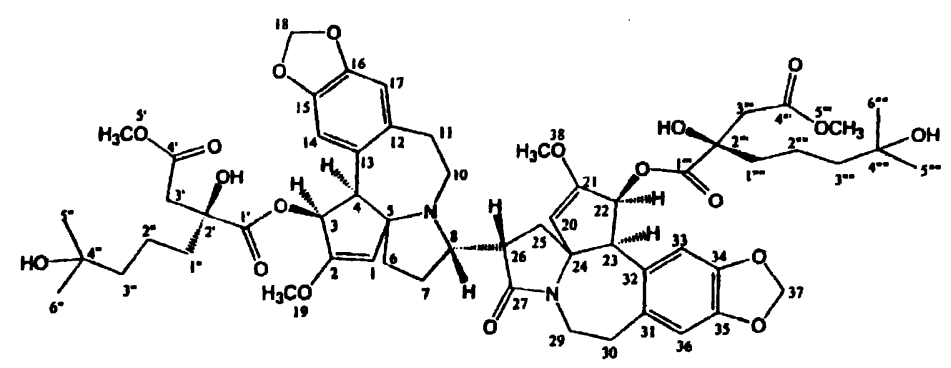


good effects in chronic granulocytic leukemia, chorionepithelioma, cancer of thymus, lymphosarcoma and Hodgkin's disease'.

Mikolajczak et al. ${ }^{14}$ isolated the alkaloid deoxyharringtonine (21) with significant antileukemic activity from C. harringtonia. It showed the antileukemic activity of the same order of magnitude as that previously reported for the other three cephalotaxine esters 39. 41 and 52. Hainanensine (38), an antitumour alkaloid from $C$. hainanensis, inhibited the growth of P388 tumour cells ${ }^{15}$.

Alkaloid G (2) and drupacine (26) are identical and showed most of the same major peaks with only minor differences in some of the reported chemical shifts. However, there are considerable differences in the melting points of alkaloid $G^{8}\left(131.0-131.5^{\circ}\right)$ and drupacine $\left(70-72^{\circ}\right)^{16}$. Alkaloids possessing the homoerythrina ring system are known to occur in Schelhammera species ${ }^{17,18}$ but from the

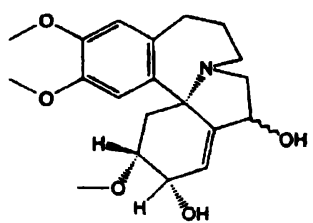

11
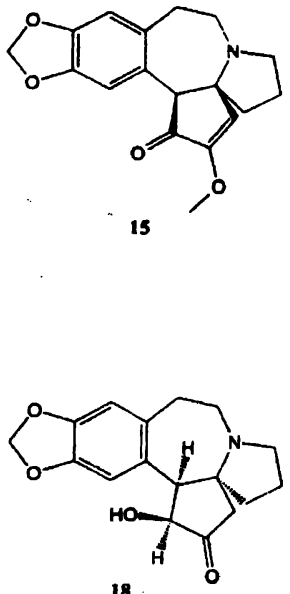

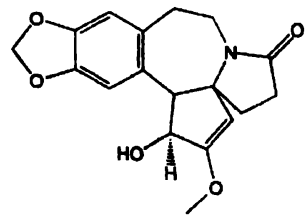

13

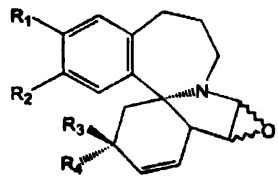

$16 R_{1}, R_{2}=-\mathrm{OCH}_{2} \mathrm{O} ; \mathrm{R}_{3}=\mathrm{OCH}_{3}, \mathrm{R}_{4}=\mathrm{H}$ $17 R_{1}, R_{2}=-O \mathrm{CH}_{2} \mathrm{O}-; R_{3}=\mathrm{H}, \mathrm{R}_{4}=\mathrm{OCH}_{3}$ $33 \mathbf{R}_{1}=\mathbf{R}_{2}=\mathrm{OCH}_{3} ; \mathrm{R}_{3}=\mathrm{OCH}_{3} ; \mathbf{R}_{4}=\mathrm{H}$ $59 R_{1}=R_{2}=\mathrm{OCH}_{3} ; R_{3}=\mathrm{H}_{1} \mathrm{R}_{4}=\mathrm{OCH}_{3}$

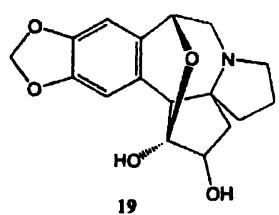

Cephalotaxus genus, for the first time, five homoerythrina alkaloids $(30,31,37,53$ and 57$)$ were isolated from C. harringtonia ${ }^{9}$. They were the simple derivatives of schelhammericine. Later on several homoerythrina alkaloids were isolated from $C$. wilsoniana and $C$. fortunei. Comosimine (16) isolated from $C$. fortunei ${ }^{19}$, epi-phelline from $C$. sinen$s i s^{20}$ and alkaloid-6 from Phelline comosa ${ }^{21}$ have the same structure. A new alkaloid drupangtonine (27) isolated from C. harringtonia showed antileukemic activity against $\mathrm{P} 388$ leukemia cells ${ }^{22}$. New cephalotaxus alkaloids bishomo-
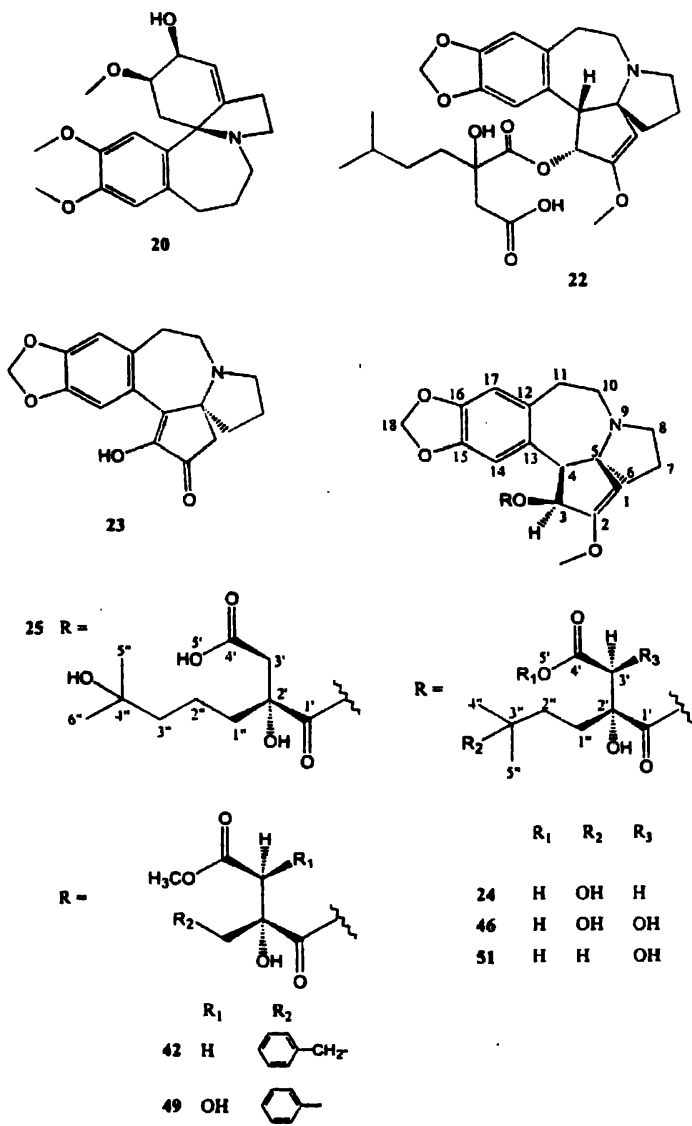

deoxyharringtonine (4), homodeoxyharringtonine (40) and nordeoxyharringtonine (56) isolated from $C$. harringtonia (var. drupacea) have shown antileukemic activity against P388 leukemia cells ${ }^{23}$. These compounds are less cytotoxic than deoxyharringtonine (21). A dimeric ester alkaloid cephalotaxidine (12) has also been isolated from C. harringtoni $a^{24}$, and this is the first example of the dimeric Cephalotaxus alkaloid. Qiu et al. $^{25}$, isolated a novel alkaloid fortunine (37) along with 11-hydroxycephalotaxine (44) and wilsonine (59) from $C$. fortunei. Alkaloids 42, 45, 47-49 and 55 showed antileukemic activities against $\mathrm{P} 388$ leukemia cells and their $\mathrm{IC}_{50}$ values were $0.28,0.38,0.33,0.17,0.19$, and $0.012 \mu \mathrm{g} / \mathrm{ml}$, respectively $^{26,27}$. Recently, Morita et $a .^{28}$ isolated potent cytotoxic alkaloids cephalezomines A-F 5-10 from $C$. harringtonia var. nana along with several known alkaloids like drupacine (26), desmethylcephalotaxine (23), cephalotaxine (14), 11-hydroxycephalotaxine (44), epi-schelhammericine B (32), 3-epi-schelhammericine (31), harringtonine (39), homodeoxyharringtonine (40), deoxyharringtonine (21) and isoharringtonine (52).

Several synthetic routes have been developed towards the synthesis of cephalotaxine (14) ${ }^{29-34}$ and its derivatives, e.g. deoxyharringtonine (21), harringtonine (39), homohar- 


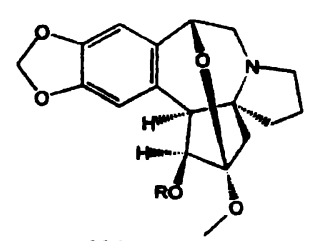

$26 \mathbf{R}=\mathbf{H}$
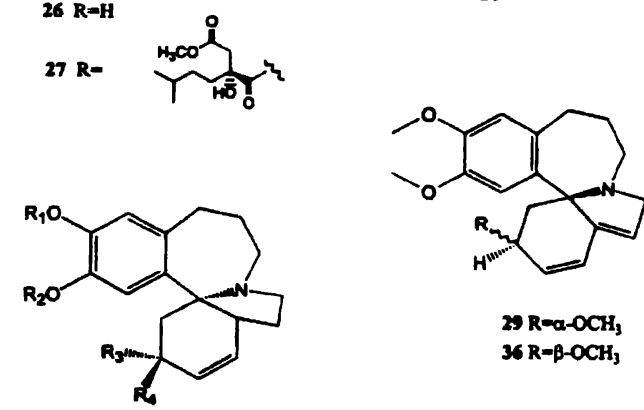

$30 R_{1}=R_{2}-C H_{3}, R_{3}-O C H_{3}, R_{1}=H$ $31 \mathrm{R}_{1}, \mathrm{R}_{2}=-\mathrm{CH}_{2}, \mathrm{R}_{3}=\mathrm{OCH}_{3}, \mathrm{R}_{4}=\mathrm{H}$ $32 \mathrm{R}_{1}=\mathrm{CH}_{3}, \mathrm{R}_{2}=\mathrm{R}_{6}-\mathrm{H}_{1} \mathrm{R}_{3}=\mathrm{OCH}_{3}$ $53 \mathrm{R}_{1}=\mathrm{R}_{2}=\mathrm{CH}_{3}, \mathrm{R}_{3} \approx \mathrm{H}_{1} \mathrm{R}_{4}=\mathrm{OCH}_{3}$ $57 \mathrm{R}_{1}=\mathrm{CH}_{3}, \mathrm{R}_{2}=\mathrm{R}_{3}=\mathrm{H}, \mathrm{R}_{4}=\mathrm{OCH}_{3}$

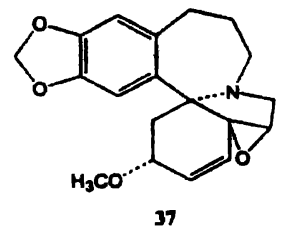<smiles>CCO[C@H]1CC2C(O)CN3CCCc4cc(OC)c(OC)cc4[C@]2(C[C@@H]1OC)C3</smiles>

34 B-OEt 35 a-OEt

37

ringtonine (41) and isoharringtonine $(52)^{35-38}$. Various alkaloids isolated from Cephalotaxus species are listed in Table 1.

\begin{tabular}{|c|c|c|}
\hline \multicolumn{3}{|c|}{ Table 1. Alkaloids from Cephalotuxus species } \\
\hline Isolated compd. & Species & Ref. \\
\hline \multirow[t]{3}{*}{ Acetylcephalotaxine (1) } & C. forrunei & $1,39.40,41.42 .43 .44$ \\
\hline & C. hainanensis & 1.45 .46 \\
\hline & C. wilsoniana & $1,8,47$ \\
\hline Alkaloid G (2) & C. wilsonianat & 8. 47 \\
\hline Anhydroharringtonine (3) & C. fortunei & 44 \\
\hline Bishomodeoxyharringtonine (4) & $\begin{array}{l}\text { C. harringtonia } \\
\text { (var. drupacea) }\end{array}$ & 23 \\
\hline Cephalezomines A-F (5-10) & $\begin{array}{l}\text { C. harringtonia } \\
\text { (var. nana) }\end{array}$ & 28 \\
\hline Cephalofortuneine (11) & C. fortunei & $1,19,48$ \\
\hline Cephalotaxidine (12) & $\begin{array}{l}\text { C. harringtonia } \\
\text { (var. drupacea) }\end{array}$ & 24 \\
\hline Cephalotaxinamide (13) & C. hainanensis & $1,45,46$ \\
\hline \multirow[t]{2}{*}{ Cephalotaxine (14) } & C. fortunei & $1,7,40.41,42,44,49,50$ \\
\hline & C. griffithii & 49 \\
\hline \multirow[t]{7}{*}{. } & C. hainanensis & $1,12,45,46$ \\
\hline & $\begin{array}{l}\text { C. harringtonia } \\
\text { (var. drupacea) }\end{array}$ & 7. $10,51,52$ \\
\hline & $\begin{array}{l}\text { C. harringtonia } \\
\text { (var. harringtonia) }\end{array}$ & $1,8.9,49,53,54,55$ \\
\hline & $\begin{array}{l}\text { C. harringtonia } \\
\text { (var. nanu) }\end{array}$ & 28 \\
\hline & C. oliveri & 1,11 \\
\hline & C. sinensis & 1. $20,56,57$ \\
\hline & C. wilsoniana & $1,8,47,49$ \\
\hline Cephalotaxinone (15) & C. fortunei & $1,40,41,53,54$ \\
\hline
\end{tabular}

\section{Comosimine (16) \\ (epi-phelline, alkaloid-6) \\ Cycloxyisoschelhammericine (17) \\ Demethylcephalotaxine (18) \\ Demethylneodrupacine (19) \\ 7-Deoxycephalofortuneıne (20) \\ Deoxyharringtonine (21)}

Deoxyharringtonic acid (22)

Desmethylcephalotaxinone (23)

Drupangtonine (27)

epi-Cephalotaxine (28)

3-epi-Fortuneine (29)

epi-Methylschelhammericine B (30)

(⿰亻)

3-epi-Schelhammericine (31)

epi-Schelhammericine B (32)

3-epi-Wilsonine (33)

2-O-Ethylcephalofortuneine (34) 2-O-Ethylepicephalofortuneine (35) Fortuneine (36)

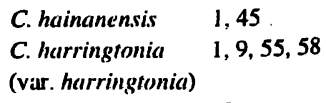

C. hainanensis $\quad 1,45$

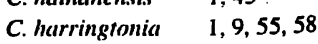

(var. harringtonia)

C. fortunei $\quad 19$

C. sinensis 20

C. hainunensis $\quad 1,45$

C. sinensis $\quad 1.20$

C. drupacea $\quad 1,51$

C. fortunei $\quad 1,40,41$

C. hainanensis $\quad 1,45,46$

C. fortunei 19

$\begin{array}{ll}\text { C. drupucea } & 23,26,27,49,52,59\end{array}$

(C. harringtonia

var. drupacea)

C. fortunci 44

C. griffithii $\quad 49,55,59$

C. hainanensis $\quad 1,12,45,55,59$

C. harringtonia $\quad 1,9,14,43,49,53$

(varr. harringtonia) 55, 58, 59, 60

C. harringtonia 28

(var. nana)

C. hainanensis $\quad 1.45,46,59,61$

C. fortunei 42

C. hainunensis $\quad 1,12,45$

C. harringtonia 55

(vitr. harringtonia)

C. harringtomia 28

(var. nana)

C. sinensis

C. hurringtonia

(var. srupucea)

C. harringtonia

(var. drupacea)

C. drupacea

$16,49.51$

C. fortunei $\quad 1,49,50$

C. griffirhii 49

C. hainanensis $\quad 1,22$

C. harringtonia 28

$\begin{array}{ll}\text { C. sinensis } & 1.20,56\end{array}$

C. wilsoniana 1.47

C. harringtonia 22

(var. drupucea)

C. fortumei

C. Inimanensis $\quad 1,12$

C. fortumei 19

C. drupacea 1

C. fortumei $\quad 53,54$

C. harringtonia 9

(var. harringtonia)

C. wilsoniana 47

C. drupacea $\quad 1.51$

C. fortumei $\quad 54,62$

C. hainanensis $\quad 1.12 .45$

C. harringtomia 1,9

(var. harringtonia)

C. harringtonia 28

(var. nemu)

C. wilsoniana $\quad 1,47$

C. drupacea $\quad 1.51$

C. hainanensis 1

C. harringtonia 1,9

(var. harringtonia)

C. harringtonia 28

(var. nama)

C. formunei

C. griffithii

C. sinensis

C. wilsoniana

C. fortunei

C. fortunei

C. fortunei

2

$40,41,53,54$

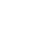


Bhattacharya et al. : Chemical constituents of Cephalotaxus species

Fortunine (37)

Hainanensine (38)

Harringtonine (39)

Homodeoxyharringtonine (40)

Homoharringtonine (41)

Homoneoharringtonine (42)

4-Hydroxycephalotaxine (43)

11-Hydroxycephalotaxine (44)

$\begin{array}{lll}\text { C. fortunei } & 25 & \\ \text { C. fortunei } & 15 & \text { Schelhammericine B (57) } \\ \text { C. hainanensis } & 1,15,45 & \\ \text { C. drupacea } & 10,27.49,52,59 & \text { Tuxodine (58) } \\ \text { C. fortunei } & 41,42,44,50,59, & \text { Wilsonine (59) } \\ & 64,65,66 & \\ \text { C. griffithii } & 49,59 & \\ \text { C. hainanensis } & 1,12.45,59 & \\ \text { C. harringtonia } & 1,9,43,53,59,60,61 . & \end{array}$

$1,9,43,53,59,60,61$

(var. harringtonia) 67.68

C. harringtonia 28

(var. nana)

C. oliveri $\quad 1,11,59$

C. sinensis $\quad 1,56,65$

C. wilsoniana $\quad 49,59$

C. harringtonia 23

(var. drupacea)

C. harringtonia $\quad 60$

(var. harringtonia)

C. harringtonia 28

(var. nana)

C. druparea

C. fortunei

C. griffinhii

C. hainamensis

C. harringtonia

(varr. harrington

C. sinensis

C. wilsoniana

C. hurringtonia

(var. drupacea)

C. fortunei

c. Arupacea

C. fortumei $\quad$ I. $25,48,49,50$

C. griffithii $\quad 1,49$

C. hurringtonia 49

(var. harringtonia)

C. harringtonia 28

(var. nuna)

11ß-Hydroxydeoxyharringtonine (45)

3'S-Hydroxy-5'-des- $O$-methylharringtonine (46)

1 $\alpha$-Hydroxyhomodeoxyharringtonine (47)

$11 \beta$-Hydroxyhomodeoxyharring-

tonine (48)

3'S-Hydroxyneoharringtonine (49)

Isocephalotaxinone (50)

Isoharringtonic acid (51)

Isoharringtonine (52)

Methylschelhammericine B (53)

Methyltaxodine (54)

Neoharringtonine (55)

Nordeoxyharringtonine (56)
C. sinensis

C. wilsoniana

C. harringtonia

(var. drupacea)

C. harringtonia

(var. drupacea)

C. harringtonia

(var. drupacea)

C. harringtonic

(var. drupaciea)

C. harringtonia

(var. drupacea)

C. fortunei

C. hainamensis

C. harringtonica

C. drupacea

C. fornunei

C. griffithii

C. huinunensis

C. harringtonia

(var hurringtonic)

C. harringtonia

(var. nema)

C. sinensis

C. wilsomiuna

1. 20,56

49

26

52

26

26

27

44. 54

1. 45.46

52

1. 27, 49. 51, 52, 59

1. $44,59,64$

49. 59

1. 12. 45,59

9. $43,53,59,60,61$.

1, 20, 59

C. harringtonia

(var. hurringtonia)

C. fortunei

C. fortunei

C. harringtonia

(var. drupacea)

C. harringtonia
1. $47,49,59$

1, 9

62

44

27

23

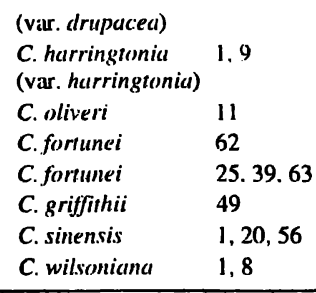

Biflavonés

Only amentoflavone (60) and its methylated derivatives have been isolated from Cephalotaxus species (Table 2). The occurrence of highly methylated derivatives of amen-
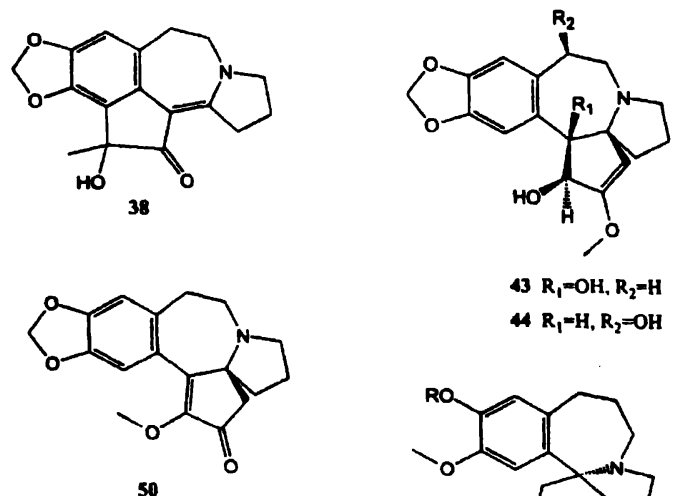

$$
\begin{aligned}
& 43 \mathrm{R}_{1}=\mathrm{OH}, \mathrm{R}_{2}=\mathrm{H} \\
& 4 \mathrm{R}_{1}=\mathrm{H}, \mathrm{R}_{2}=\mathrm{OH}
\end{aligned}
$$<smiles>[R6]Oc1cc2c(cc1OC)[C@]13CCCN2CCC1=CC[C@@H](OC)C3</smiles>

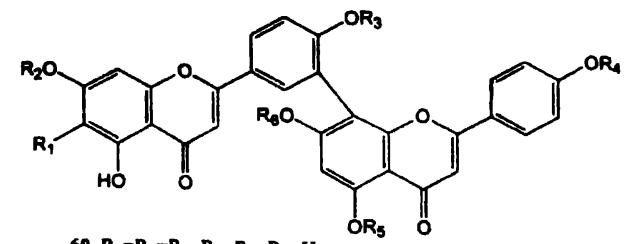

$R_{1}=R_{2}=R_{3}=R_{4}=R_{5}=R_{6}=H$

$61 R_{1}=R_{5}=H ; R_{2}=R_{3}=R_{4}=R_{6}=C_{3}$

$62 R_{1}=R_{2}=R_{4}=R_{5}=R_{6}=H_{;} R_{3}=C H_{3}$

$63 R_{1}=R_{4}-R_{5}=R_{6}=H_{;} R_{2}=R_{3}=C_{3}$

A $R_{1}=R_{2}=R_{5}=H ; R_{3}=R_{4}=R_{6}=C_{3}$

$65 R_{1}=R_{2}=C_{3} ; R_{3}=R_{4}=R_{3}=R_{6}=H$

$66 R_{1}=H ; R_{2}=R_{3}=R_{4}=R_{3}=R_{6}=C_{3}$

$67 R_{1}=R_{5}=R_{6}=H_{;} R_{2}=R_{3}=R_{4}=C_{3}$

$68 R_{1}=R_{3}=R_{1}=R_{9}=R_{6}=H_{;} R_{2}=C H_{3}$

$6 R_{1}=R_{2}=R_{3}-C H_{3} ; R_{4}-R_{3}-R_{6}=H$

toflavone, i.e. tetra- $O$-methylamentoflavone (61) was first reported by Ishratullah et al. ${ }^{72}$ from $C$. harringtonia. 6-CMethyl-7- $O$-methylamentoflavone (65) isolated from $C$. harringtonia was the first example of a naturally occurring biflavone bearing a $C$-methyl group on a flavone nucleus ${ }^{73}$. Another biflavone bilobetin (62) has been isolated from $C$. koreana nakai by Yook et al. ${ }^{74}$ along with other biflavones, i.e. amentoflavone- $4^{\prime}, 4^{\prime \prime \prime}, 7^{\prime}, 7^{\prime \prime \prime}$-tetramethyl ether (61), sciadopitysin (67) and ginkgetin (63). Recently, Kuo et al. ${ }^{75}$ have reported a novel cytotoxic $C$-methylated biflavone 
taiwanhomoflavone (69) from $C$. wilsoniana. Different biflavones isolated from Cephalotaxus species are listed in Table 2.

\begin{tabular}{|c|c|c|}
\hline \multicolumn{3}{|c|}{ Table 2. Biflavones from Cephalotaxus species } \\
\hline Isolated compd. & Species & Ref. \\
\hline \multirow[t]{3}{*}{ Amentoflavone (60) } & C. drupacea & 76 \\
\hline & C. griffithii & 77 \\
\hline & $\begin{array}{l}\text { C. harringtonia } \\
\text { (var. harringtonia) }\end{array}$ & 72. 73 \\
\hline \multirow[t]{3}{*}{$\begin{array}{l}\text { Amentoflavone- } 4^{\prime}, 4^{\prime \prime \prime} \cdot 7^{\prime}, 77^{\prime \prime} \text { - } \\
\text { tetramethyl ether (61) }\end{array}$} & $\begin{array}{l}\text { C. fortunei } \\
\text { C. Maringtonia } \\
\text { (var. hurringtonial) }\end{array}$ & $\begin{array}{l}78 \\
7273\end{array}$ \\
\hline & C. koreana nakai & 74 \\
\hline & C. oliveri & 79 \\
\hline Bilobetin (62) & C. koreana nakai & 74 \\
\hline \multirow[t]{4}{*}{ Ginkgetin (63) } & C. drupacea & 76 \\
\hline & C. griffithi & 77 \\
\hline & $\begin{array}{l}\text { C. harringtonia } \\
\text { (var. harringtonia) }\end{array}$ & 72 \\
\hline & C. koreana nakai & 74 \\
\hline \multirow[t]{2}{*}{ Kayaflavone (64) } & C. drupacea & 80 \\
\hline & $\begin{array}{l}\text { C. harringtonia } \\
\text { (var. harringtonia) }\end{array}$ & 72 \\
\hline $\begin{array}{l}\text { 6- } C \text {-Methyl-7-O-methyl- } \\
\text { amentoflavone (65) }\end{array}$ & $\begin{array}{l}\text { C. griffithii } \\
\text { C. harringtonial }\end{array}$ & $\begin{array}{l}77 \\
73,81\end{array}$ \\
\hline Oliveriflavone (66) & C. oliveri & 79 \\
\hline \multirow[t]{5}{*}{ Sciadopitysin (67) } & C. drupacea & 76 \\
\hline & C. firrunei & 78 \\
\hline & $\begin{array}{l}\text { C. harringtomia } \\
\text { (vir. harringtonia) }\end{array}$ & 72 \\
\hline & C. koreana nakai & 74 \\
\hline & C. oliveri & 79 \\
\hline \multirow[t]{4}{*}{ Sequoiaflavone (68) } & C. arupacea & 76 \\
\hline & C. griffithii & 77 \\
\hline & $\begin{array}{l}\text { C. harringtonia } \\
\text { (var. drupacea) }\end{array}$ & 73 \\
\hline & $\begin{array}{l}\text { C. harringtonia } \\
\text { (var. harringtonia) }\end{array}$ & 72 \\
\hline Taiwanhomoflavone-A (69) & C. wilsoniana & 75 \\
\hline
\end{tabular}

\section{Flavones}

Various flavones isolated from different Cephalotaxus species are compiled in Table 3.

Table 3. Flavones from Cephalotuxus species

\begin{tabular}{lll} 
Isolated compd. & Species & Ref. \\
Apigenin (70) & C. griffithii & 77 \\
Cephalotaxoside (71) & C. drutucea & 82 \\
Chrysoeriol (72) & C. fornunei & 83 \\
Kaempferol-3-O-rutinoside (73) & C. follicularis & 84 \\
Luteolin-7-O-glucoside (74) & C. follicularis & 84 \\
Myricetin-3-O-glucoside (75) & C. follicularis & 84 \\
Myricetin-3-O-rutinoside (76) & C. follicularis & 84 \\
Quercetin (77) & C. griffithii & 82 \\
Quercetin-3-O-glucoside (78) & C. follicularis & 84 \\
Quercetin-3-O-rhamnoside (79) & C. follicularis & 84 \\
& C. griffithii & 77 \\
Quercetin-3-O-rutinoside (80) & C. follicularis & 84 \\
\hline
\end{tabular}

\section{Sterols}

Most of the sterols occurring in Cephalotaxus species have been isolated from C. drupacea ${ }^{85}$ (Table 4). Among them, $\beta$-sitosterol has been found to be present in abundance. We have isolated withaferin A (81) from C. griffithil $^{86}$. This is the first report of the occurrence of<smiles>[R6]c1cc([R2])c2c(=O)c([R2])c(-c3cc([R9])c(O)c([R6])c3)oc2c1</smiles>

$70 R_{1}=R_{4}=R_{3}=H ; R_{2}=R_{3}=O H$

$71 R_{1}=R_{4}=R_{3}=H ; R_{2}=0$-thamnosyl; $R_{3}=O H$

$72 R_{1}=R_{5}=H ; R_{2}=R_{3}=O H ; R_{4}=\mathrm{OCH}_{3}$ $73 R_{1}=$ O-rutinosyl; $R_{2}=R_{3}=O H ; R_{4}=R_{5}=H$

$74 R_{1}=R_{5}=H ; R_{2}=R_{4}=O H ; R_{3}=0$-glucosyl

$75 R_{1}=0$-glucosyl; $R_{2}=R_{3}=R_{4}=R_{5}=O H$

$76 R_{1}=$ O-rutinosyl; $R_{2}=R_{3}=R_{4}=R_{5}=O H$

$77 R_{1}=R_{2}=R_{3}=R_{4}=O H ; R_{5}=H$

$78 R_{1}=$ O-glucosyl; $R_{2}=R_{3}=R_{4}=O H ; R_{5}=H$

$79 R_{1}=$ O-rhamnosyl; $R_{2}=R_{3}=R_{4}=O H ; R_{5}=H$

$80 R_{1}=$-rutinosyl; $R_{2}=R_{3}=R_{4}=O H ; R_{5}=H$

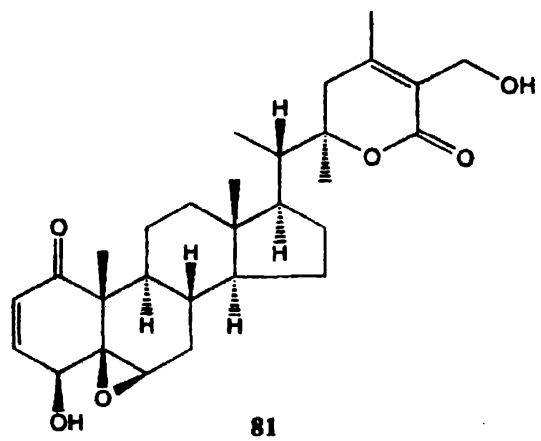

withaferin A (81) from the family and it has been reported to possess significant inhibitory activity against sarcoma 180 (tumour), sarcoma black and the mammary adenocarcinoma in mice ${ }^{87}$ and also antifungal activity ${ }^{88}$.

\begin{tabular}{lll}
\hline \multicolumn{2}{c}{ Table 4. Sterols isolated from Cephalotaxus species } \\
Isolated compd. & Species & Ref. \\
Campesterol & C. drupacea & 85 \\
Cholesterol & C. drupacea & 85 \\
24-Methylenecholesterol & C. drupacea & 85 \\
B-Sitosterol & C. drupacea & 85 \\
& C. griffithii & 86 \\
Stigmasterol & C. drupacea & 85 \\
Withaferin A (81) & C. griffithii & 86 \\
\hline
\end{tabular}

\section{Miscellaneous compounds}

Besides alkaloids, flavones, bisflavones, sterols, a few other class of compounds, i.e. taxanes, fatty acids, carbohydrates, lactones have also been isolated from different Cephalotaxus species.

Harringtonolide (85), a plant growth inhibitory tropone, was isolated from $C$. harringtonia by Buta et al. ${ }^{89}$. An an- 


\section{Bhattacharya et al. : Chemical constituents of Cephalotaxus species}

titumour compound, hainanolide (83) was isolated for the first time from $C$. hainanensis ${ }^{45.90}$ and later on from $C$. fortunei $^{91}$ and $C$. sinensis ${ }^{20}$. Hainanolide in tissue culture was noticed to have a plaque formation inhibiting activity against influenza virus type A (WS), Newcastle disease virus, Jap. B encephalities virus ${ }^{86}$ and vaccina virus ${ }^{92}$. Chen and $\mathrm{Luo}^{93}$ isolated novel 10-deacetylbaccatin-III (82) from the leaves of $C$. sinensis.

Table 5. Miscellaneous compounds from Cephalotaxus species

\begin{tabular}{lll} 
lsolated compd. & Species & Ref. \\
10-Deacetylbuccatin-III (82) & C. sinensis & 93 \\
Hainanolide (83) & C. fortunei & 91 \\
& C. hainumensis & 45.90 \\
Hainunolidol (84) & C. sinensis & 20,94 \\
Harringtonolide (85) & C. hainancensis & $39.46 .90,95$ \\
& C. hurringtonia & 89 \\
meso--nositol & (var. harringtomia) & \\
Linoleic acid & C. fortunei & 41 \\
Oleic acid & C. drupacea & 85 \\
P.lmitic acid & C. drupacea & 85 \\
Sucrose & C. drupacea & 85 \\
Fortunolides A (86) & C. griffithii & 86 \\
Fortunolides B (87) & C. forrunei & 39 \\
\hline
\end{tabular}
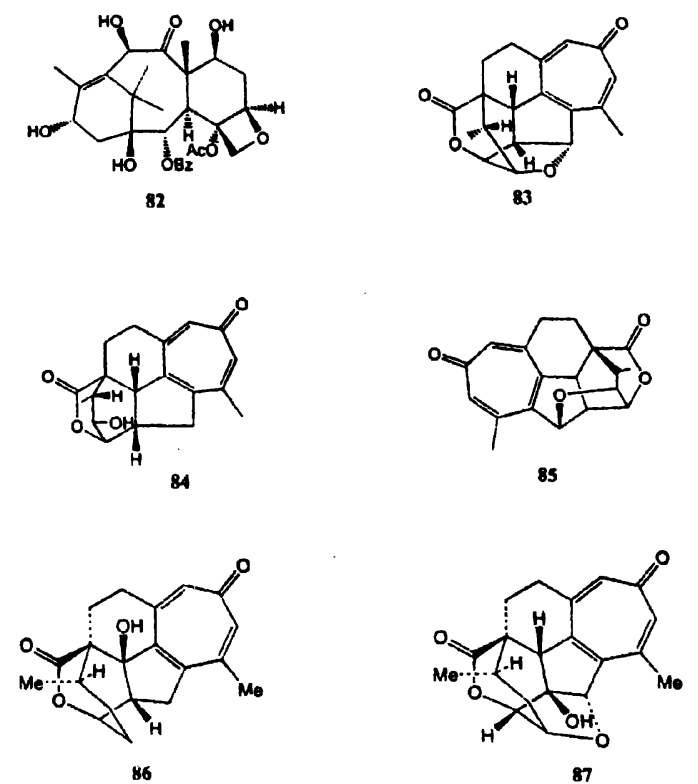

Several fatty acids have been isolated from C. drupacea. The major fatty acids were unsaturated ones which predominated over the saturated ones. Oleic and linoleic acids were the main unsaturated compounds and palmitic acid was the most abundant saturated fatty acid85. meso-Inositol, a sugar, was ișolated from C. fortunei41. Du et al.39 isolated two new lactones, fortunolides A (86) and B (87) alongwith compounds, hainanolidol (84), acetyl cephalotaxine (1) and wilsonine (59) from C. fortunei.

\section{Conclusion}

The Cephalotaxus genus is found to contain mainly alkaloids with typical carbon skeleton and having interesting and potent antitumour and antileukemic properties, besides a number of biflavones, flavones, taxanes, sterols and acids. The unique structures and potent antileukemic activities of the cephalotaxine and homoerythrina alkaloids of this genus, have drawn attention of many chemists. Our endeavour will help to locate the comprehensive information on this genus.

\section{Acknowledgement}

Financial support from C.S.I.R. and U.G.C., New Delhi, is highly acknowledged.

\section{References}

1. L. Huang and Z. Xue in "The Alkaloids : Chemistry and Physiology", cd. A. Brossi, Academic, London, 1984, Vol. 23.

2. "The Wealth of India : Raw Materials", PID-CSIR, New Delhi, 1992, Vol. 3, p. 437.

3. W. Cheng. L. Fu and C. Chao, "Flora Reipublicae Popularis Sinicae" (Delectis Florae Reipublicae Popularis Sinicae, Agendac Acaderniae Sinicae Edita), Beijing, 1978, Vol. 7, p. 423.

4. Y. H. Chen and G. Huang, Zhongcaoykeo Tongxun, 1977, 254; Ref. 1.

5. H. M. Chang and P. P. H. But, "Pharmacology and Applications of Chinese Materia Medica", World Scientific, Singapore, 1989, Vol. 1, p. 54.

6. M. E. Wall, C. R. Eddy, J. J. Willaman, D. S. Correll, B. G. Schubert and H. S. Gentry. J. Am. Pharm. Assoc., 1954, 43. 503 (Chem. Abstr., 1954, 48. 13167).

7. W. W. Paudler, G. I. Kerley and J. Mckay, J. Org. Chem.. 1963, 28, 2194.

8. R. G. Powell, K. L. Mikolajczak, D. Weisleder and C. R. Smith. Jr., Phytochemistry. 1972, 11. 3317.

9. R. G. Powell, Phytochemistry: 1972. 11, 1467.

10. R. G. Powell, D. Weisleder, C. R. Smith. Jr. and I. A. Wolff, Tetrahedron Lett., 1969, 4081

11. F. X. Zhang. Z. H. Wang, W. D. Pan, Y. J. Li, L. T. Mai, J. Q. Sun and G. E. Ma, Chilh Wu Hsteh Pao. 1978, 20. 129 (Chem. Absir., 1978, 89. 190990).

12. Chinese Academy of Medical Sciences. Hua Hsueh Hsueh Pao, 1976. 34, 283 (Chem. Abstr., 1978, 88. 126256).

13. S. Takeda, N. Yajima, K. Kitazato and N. Unemi, J. PharmacobioDyn., 1982, 5. 841 (Chem. Alsstr., 1983. 98, 27435).

14. K. L. Mikolajczak, R. G. Powell and C. R. Smith, Jr., Tetrahedron. 1972. 28, 1995.

15. N. C. Sun and H. T. Liang, Yao Hsueh Tung Pao, 1980. 15, 39 (Chem. Abstr., 1981, 95. 175614).

16. R. G. Powell, R. V. Madrigal, C. R. Smith, Jr. and K. L. Mikolajczak, J. Org. Chem., 1974. 39, 676.

17. J. S. Fitzgerald, S. R. Johns, J. A. Lamberton and A. A. Sioumis, Aust. J. Chem., 1969, 22. 2187. 


\section{J. Indian Chem. Soc., Vol. 79, October 2002}

18. S. R. Johns, J. A. Lamberton, A. A. Sioumis and H. Suares, Aust. J. Chem., 1969, 22, 2203.

19. G. Ma, J. Chen, D. Wang and H. Huang, Zhiwu Xuebao, 1989, 31 , 137 (Chem. Abstr., 1990, 113, 74722).

20. L. Ren and Z. Xue, Zhongcaoyao, 1981, 12, 1 (Chem. Abstr., 1982, 96, 3672).

21. N. Langlois, B. C. Das, P. Potier and L. Lacombe, Bull. Soc. Chim. Fr., 1970, 3535.

22. I. Takano, I. Yasuda, M. Nishijima, Y. Hitotsuyanagi, K. Takeya and H. Itokawa, Biorg. Med. Chem. Lett., 1996, 6, 1689.

23. I. Takano, I. Yasuda, M. Nishijima, Y. Hitotsuyanagi, K. Takeya and H. Itokawa. J. Nat. Prod., 1996, 59, 965.

24. I. Takano, I. Yasuda, M. Nishijima, Y. Hitotsuyanagi, K. Takeya and H. Itokawa, Tetrahedron Lett., 1996, 37, 7053.

25. M. Qiu, B. Lu, X. Ma and R. Nei. Yınnan Zhiwu Yanjiu, 1997, 19, 97 (Chem. Abstr.. 1997, 127, 275355).

26. I. Takano, I. Yasuda, M. Nishijina, Y. Hitotsuyanagi, K. Takeya and H. Itokawa, J. Nat. Prod., 1996, 59, 1192.

27. I. Takano, I. Yasuda, M. Nishijima. Y. Yanagi, K. Takeya and H. Itokawa, Phytochemistry. 1997, 44. 735.

28. H. Morita, M. Arisaka, N. Yoshida and J. Kobayashi. Tetrahedron, 2000, 56, 2929.

29. J. Anerbach and S. M. Weinreb, J. Am. Chem. Soc., 1972, 94, 7172.

30. S. M. Weinreb and J. Anerbach, J. Am. Chem. Soc., 1975, 97. 2503.

31. L. J. Dolby, S. J. Nelson and D. Senkovich, J. Org. Chem., 1972, 37, 3691.

32. I. Tse and V. Snieckus, J. Chem. Soc., Chem. Commun., 1976, 505.

33. B. Weinstein and A. R. Craig. J. Org. Chem., 1976. 41, 875.

34. M. F. Semmelhack, B. P. Chong and C. D. Jones, J. Am. Chem. Soc., 1972. 94, 8629.

35. T. T. Harding, J. N. Vllrich. F. T. Chiu, S. F. Chen and P. S. Mariana, J. Org. Chem., 1982, 47, 3360.

36. S. Li and J. Dai, Acta Chin. Sin., 1975, 33, 75.

37. K. L. Mikolajczak and C. R. Smith, Jr., J. Org. Chem., 1978, 43, 4762.

38. Z. Zhao, Y. Xi, H. Zhao, J. Hou, J. Zhang and Z. Wang, Acta Pharm. Sin., 1980, 15, 46.

39. J. Du, M. H. Chiu and R. L. Nie, J. Nat. Prod., 1999, 62, 1664 (Chem. Abstr., 2000, 132. 33203).

40. W. W. Paudler and J. Mckay, J. Org. Chem. 1973, 38, 2110.

41. W. Yin, Linchan Huaxue Yu Gingyl, 1986, 6, 36 (Chem. Abstr., 1986. 105, 222807).

42. G. E. Ma, L. T. Lin, T. Y. Chao and H. C. Fan, Hua Hsueh Hsueh Pao, 1977. 35, 201. (Chem. Alsstr. 1979, 90, 19013).

43. D. Z. Wang, G. E. Ma and R. S. Xu, Yaoxue Xuebao, 1992, 27, 178 (Chem. Abstr., 1992, 117 163421).

44. D. Z. Wang. G. E. Ma and R. S. Xu. Yaoxue Xuebao, 1992, 27, 173 (Chem. Alstr. 1992, 117, 86698).

45. Z. Xue, N. J. Sun, D. H. Chen, L. Z. Xu, W. M. Chen, L. J. Ren, X. T. Liang and L. Huang. Chem. Abstr., 1983, 98, 194936.

46. Z. Xue, L. Xu, D. H. Chen and L. Huang, Acta Pharm. Sin., 1981.
16, 752 (Chem. Abstr., 1982, 96. 82690).

47. H. Furukawa, M. Itoigawa, M. Haruna, Y. Jinno. K. Ito and S. T. Lu, Yakugaku Zasshi, 1976, 96, 1373 (Chem. Abstr., 1977, 86. 103043).

48. G. E. Ma, L. T. Lin, T. Y. Chao and H. C. Fan, Hua Hsueh Hsueh Pao, 1978, 36, 129 (Chem. Abstr., 1978, 89, 193837).

49. G. F. Spencer, R. D. Plattner and R. G. Powell J. Chromatogr., 1976, 120, 335.

50. G. Ma, C. Lu and G. Fan, Yaoxue Tongbao, 1982, 17, 205 (Chem. Abstr., 1982, 97, 123909).

51. S. Asada, Yakugaku Zasshi, 1973, 93, 916 (Chem. Abstr., 1973, 79, 123699).

52. I. Takano, I. Yasuda, M. Nishijima, Y. Hitotsuyanagi, K. Takeya and H. Itokawa, Phytochemistry, 1996, 43, 299.

53. R. G. Powell, D. Weislder and C. R. Smith, Jr., J. Pharm. Sci., 1972, 61, 1227.

54. W. Lin. R. Chen and Z. Xue, Yaoxue Xuebao, 1985, 20, 283 (Chem. Abstr., 1985, 103, 85049).

55. R. G. Powell and K. L. Mikolajczak, Phytochemistry, 1973, 12. 2987.

56. Academia Sinica Institute of Botany, Chih Wu Hseuh Pao, 1980, 22, 156 (Chem. Abstr., 1980, 93, 235142).

57. H. He, Znongcaoyao, 2001, 32, 201 (Chem. Abstr., 2001, 135, 254452).

58. R. J. Parry, M. N. T. Michael, J. M. Schwab and B. M. Foxman, J. Am. Chem. Soc. 1980, 102, 1099.

59. X. Lu, S. Zhang, Z. Wang and M. Wang, Yaoxue Xuebuo, 1981, 16, 773 (Chem. Abstr., 1982, 96, 91706).

60. N. E. Delfel and J. A. Rothfus, Phytochemistry, 1977, 16, 1595.

61. R. G. Powel and C. R. Smith, Jr., Chem. Abstr., 1974, 80, 112615.

62. G. Ma, D. Wang, J. Chen and H. Huang, Huaxue Xuebao, 1989, 47, 1128 (Chem. Abstr., 1990, 113, 55803).

63. G. E. Ma, C. Lu, H. N. El-Sohly, M. A. El-Sohly and C. E. Turner, Phytochemistry, 1983, 22, 251.

64. D. Cai and M. Gu. Zhongguo Yiyao Gongye Zazhi, 1991, 22, 72 (Chem. Abstr., 1991, 115, 57275).

65. Y. Ma, M. Guo, T. Zhu. G. Ma, C. Lu, H. Huang and Y. Yang, Zhiwu Xuebao, 1984, 26, 405 (Chem. Abstr., 1984, 101, 207668).

66. Y. G. Liu, Chem. Abstr., 1990, 113, 197998.

67. R. G. Powell and C. R. Smith, Jr.. Chem. Abstr., 1975, 83, 33023.

68. R. G. Powell, S. P. Rogovin and C. R. Smith, Jr., Chem. Abstr., 1974, 81, 41323.

69. C. R. Smith Jr., R. G. Powell and K. L. Mikolajczak, Cancer Treat. Rep., 1976. 60, 1157 (Chem. Abstr., 1976, 85, 198065).

70. R. J. Parry, D. D. Sternbach and M. D. Cabelli, J. Am. Chem. Soc., 1976, 98, 6380.

71. G. Ma, G. Q. Sun, M. A. El-Sohly and C. E. Turner, J. Nat. Prod., 1982. 45, 585 (Chem. Abstr., 1983, 98, 68815).

72. K. Ishratullah, W. Rahman, M. Okigawa and N. Kawano, Indian J. Chem., Sect. B, 1981, 20, 935.

73. M. Aqil, W. Rahman, N. Hasaka, M. Okigawa and N. Kawano, $J$. Chem. Soc., Perkin Truns. I, 1981. 1389.

74. C. S. Yook, J. H. Jung, J. H. Jeong, T. Nohara and S. Y. Chang, 


\section{Bhattacharya et al. : Chemical constituents of Cephalotaxus species}

Nat. Prod. Sci., 2000, 6, 1 (Chem. Abstr., 2000, 133, 101987).

75. Y. H. Kuo, C. H. Lin, S. Y. Hwang, Y. C. Shen, Y. C. Lee and S. Y. Li, Chem. Pharm. Bull., 2000, 48, 2000.

76. N. U. Khan. M. Ilyas, W. Rahman, M. Okigawa and N. Kawano, Phytochemistry, 1971, 10, 2541.

77. M. Kamil, N. A. Khan, I. Ahmad, M. Ilyas and W. Rahman, J. Indian Chem. Soc., 1982, 59, 1199.

78. Z. Ma, G. He and W. Yin, Zhiwu Xuebao, 1984, 26, 416 (Chem. Abstr., 1985, 102, 3254).

79. Z. Ma, G. He and W. Yin, Zhiwu Xuebao, 1986, 28, 641 (Chem. Abstr., 1987, 106, 135286).

80. W. Baker and W. D. Ollis in "Recent Developments in the Chemistry of Natural Phenolic Compounds", ed. W. D. Ollis, Pergamon. New York, 1961, p. 181.

81. M. Aqil, W. Rahman, M. Okigawa and N. Kawano, Chem. Ind. (London), 1976, 13, 567 (Chem. Abstr., 1977, 86, 5273).

82. V. Plouvier, C. R. Habd. Seances Acad. Sci., Ser: D. 1966, 263, 1529 (Chem. Abstr., 1967, 66. 55708).

83. W. Yang, Y. Xing, J. Xu and X. Shi, Lanzhou Daxue Xuebao Ziran Kexueban 1983, 19, 114 (Chem. Abstr., 1983, 98, 212876).

84. K. W. Nicholls, B. A. Bohm and R. Ornduff, Biochem. System. Ecol., 1985, 13, 261 (Chem. Abstr., 1985, 103, 157401).

85. R. Cerri, A. Dini, V. DeFeo and F. Senatore, Herba Pol., 1986, 32.
81 (Chem. Abstr., 1988, 108, 34834).

86. Unpublished results from our laboratory.

87. B. Shohat, S. Gitter, A. Abraham and D. Lavie, Cancer Chemother. Rep., 1967, 51, 271 (Chem. Abstr. 1967, 67, 105691).

88. "The Merck Index", 9th. ed., ed. M. Windholz, Merck Co. Inc., Rahway, 1976, 9716.

89. J. G. Buta, J. L. Flippen and W. R. Lusby, J. Org. Chem., 1978, 43, 1002.

90. N. J. Sun, Z. Xue, X. T. Liang and L. Huang. Yao Hsueh Hsueh Pao, 1979, 14, 39 (Chem. Abstr., 1980, 92, 220590).

91. N. J. Sun, Z. F. Zhao. R. T. Chen, W. Lin and Y. Z. Zhou, Yao Hsueh Hsueh Pao, 1981, 16. 233 (Chem. Abstr., 1981, 95, 156431).

92. K. Shuging, C. Shengyong and T. Li, Acta Pharm. Sin., 1981, 16, 867.

93. C. Chen and S. Luo, Zhongguo Yiyao Gongye Zazhi, 1997, 28, 375 (Chem. Abstr.. 1998, 128, 319317).

94. G. Bao, C. He and C. Xu, Zhongguo Yixue Kexueyuan Xuebao, 1983, 5, 89 (Chem. Abstr., 1984, 100, 59893).

95. Z. Xue, N. Sun and X. Liang, Yaoxue Xuebao, 1982, 17, 236 (Chem. Abstr., 1982, 96, 177999). 